\title{
Musashi2 promotes EGF-induced EMT in pancreatic cancer via ZEB1-ERK/MAPK signaling
}

Weiwei Sheng ${ }^{1 \dagger}$, Xiaoyang Shi ${ }^{1 \dagger}$, Yiheng Lin ${ }^{1}$, Jingtong Tang ${ }^{1}$, Chao Jia ${ }^{1}$, Rongxian Cao ${ }^{2}$, Jian Sun ${ }^{1}$, Guosen Wang $^{3}$, Lei Zhou ${ }^{4}$ and Ming Dong ${ }^{1^{*}}$ (D)

\begin{abstract}
Background: Our previous study showed Musashi2 (MSI2) promoted chemotherapy resistance and pernicious biology of pancreatic cancer (PC) by down-regulating Numb and p53. We further explored the novel molecular mechanism involving its oncogenic role in PC development.

Methods: We investigated the potential role and mechanism of MSI2 in EGF-induced EMT in PC in vitro and vivo. Results: EGF enhanced EGFR (epidermal growth factor receptor) phosphorylation, induced EMT and activated ZEB1ERK/MAPK signaling in 2 PC cells. However, MSI2 silencing reversed EGF stimulated function, including inhibiting EGF-promoted EMT-like cell morphology and EGF-enhanced cell invasion and migration. Meanwhile, MSI2 silencing inhibited EGF-enhanced EGFR phosphorylation at tyrosine 1068 and reversed EGF-induced change of the key proteins in EMT and ZEB1-ERK/MAPK signaling (ZEB1, E-cad, ZO-1, $\beta$-catenin, pERK and c-Myc). Additionally, MSI2 was co-stained and co-immunoprecipitated with ZEB1, pERK and c-Myc in PC cells by IF and co-IP, implying a close interaction between them. In vivo, MSI2 silencing inhibited pancreatic tumor size in situ and distant liver metastases. A close relationship of MSI2 with EMT and ZEB1-ERK/MAPK signaling were also observed in vivo and human $P C$ samples, which coordinately promoted the poor prognosis of $P C$ patients.

Conclusions: MSI2 promotes EGF-induced EMT in PC via ZEB1-ERK/MAPK signaling.
\end{abstract}

Keywords: Musashi2, EGF, ZEB1, ERK/MAPK pathway, C-Myc, Epithelial mesenchymal transition, Pancreatic cancer

\section{Background}

Pancreatic cancer (PC) is one of the most malignant tumors in the world. From 2000 to 2011, the incidence rate and age-standardized mortality of PC in Chinese men have greatly increased, ranking the top first and second among malignant tumors [1]. In 2018, about 55,440 people were diagnosed with and about 44,340 died of PC in United States [2]. PC is now predicted to become the second cancer related death in 2020 [3]. Aggressive local invasion and distant metastase contribute to the poor prognosis of PC patients which is significantly promoted by epithelial-mesenchymal transition (EMT) [4]. PC

\footnotetext{
* Correspondence: dongming@cmu.edu.cn

tWeiwei Sheng and Xiaoyang Shi contributed equally to this work.

${ }^{1}$ Department of Gastrointestinal Surgery, the First Hospital, China Medical

University, Shenyang 110001, China

Full list of author information is available at the end of the article
}

stimulated by EMT loses epithelial character, gains strong invasive mesenchymal cells, and finally transforms into the highly malignant phenotype $[2,5]$.

Musashi RNA-binding protein 2 (MSI2) is a translational repressor identified in Drosophila melanogaster. It plays a significant role in regulating asymmetric division, stemness maintenance and neural differentiation, hematopoietic and gastrointestinal systems in many species [6-9]. Recently, MSI2 dysregulation has been reported in various hematology and solid tumors, including acute and chronic myeloid leukemia (AML and CML), brain, lung, gastric, hepatocellular and bladder cancers [10-15]. Our previous study first showed MSI2 overexpression promoted cell invasion and metastasis in PC by down-regulating Numb in vivo and vitro [16]. Additionally, under gemcitabine or cisplatin treatment, MSI2 promoted chemotherapy resistance and pernicious biology of PC in p53-dependent manner [17]. In 
current study, we intend to investigate the novel signaling involving the oncogenic role of MSI2 in advanced progression of PC. We first found MSI2 promoted EGF-induced EMT in PC through ZEB1-ERK/MAPK signaling in vitro and vivo, which has not been reported previously, to our knowledge.

\section{Materials and methods}

\section{Tissue samples and cell lines}

This study was approved by the academic review board of the first hospital of China Medical University and the approval in consent form that was signed by each resident patient. Seventy four paraffin-embedded ductal adenocarcinoma samples were obtained from postoperative patients at the First Hospital of China Medical University between 2010 and 2015 with complete follow up data. AsPC-1, BxPC-3, PANC-1, Miapaca-2 and SW1990 human PC cell lines were purchased from the Cell Bank of the Chinese Academy of Sciences (Shanghai, China). These cell lines were maintained in recommended growth media with $10 \%$ fetal calf serum (Gibco Invitrogen, Carlsbad, CA).

\section{Immunofluorescence (IF) staining}

PC cells were plated into 24-well culture plates, fixed in $4 \%$ paraformal dehyde, permeabilized with $0.25 \%$ Triton X-100 and incubated with 5\% BSA. Then the slices were stained with the primary antibodies: MSI2 (Abcam, Cambridge, UK), ZEB1 (Proteintech, Chicago, IL, USA), pERK (Cell signaling technology, MA, USA) and c-Myc (Proteintech). The secondary antibodies were conjugated with FITC and TRITC (Vector laboratories, California, USA). Cells were then co-stained with blue Hoechest33258 (Vector laboratories) for nuclear visualizing and detected under microscope (Nikon Microphot-FX, Japan).

\section{Immunohistochemistry}

As described previously [18], 4-um sections were covered with $0.3 \% \mathrm{H}_{2} \mathrm{O}_{2}$, subjected to high pressure and added with $10 \%$ normal goat serum. Then the slices were incubated with primary antibodies: MSI2 (Abcam), ZEB1 (Proteintech), E-cadherin(E-cad, Abcam), c-Myc (Proteintech), pERK (Cell signaling technology), ZO-1 (Proteintech), $\beta$-catenin (Proteintech) and Vimentin (Proteintech). After 3 times wash in PBS, slices were incubated with the secondary antibodies, treated with streptavidin-peroxidase reagent, visualized with $\mathrm{DAB}$, counterstained with haematoxylin and detected under microscope. Staining intensity was scored as $0-3$ (negative, weak, medium and strong). Extent of staining was scored as 0 (<5\%), 1 (5-25\%), 2 (26-50\%), 3 (51-75\%), and $4(>75 \%)$ according to the positive staining areas to the whole carcinoma. The final scores were calculated by three pathologists. The same score evaluation was used in both human PC samples and in vivo.

\section{Western blot}

Whole protein lysates were prepared from sg1-MSI2, sg2MSI2, MSI2-GFP, MSI2-GFP plus MSI2siRNA and scramble transfected PC cell lines with or without EGF (50 ng/ $\mathrm{ml})$. Samples were loaded onto $10 \%$ SDS-polyacrylamide
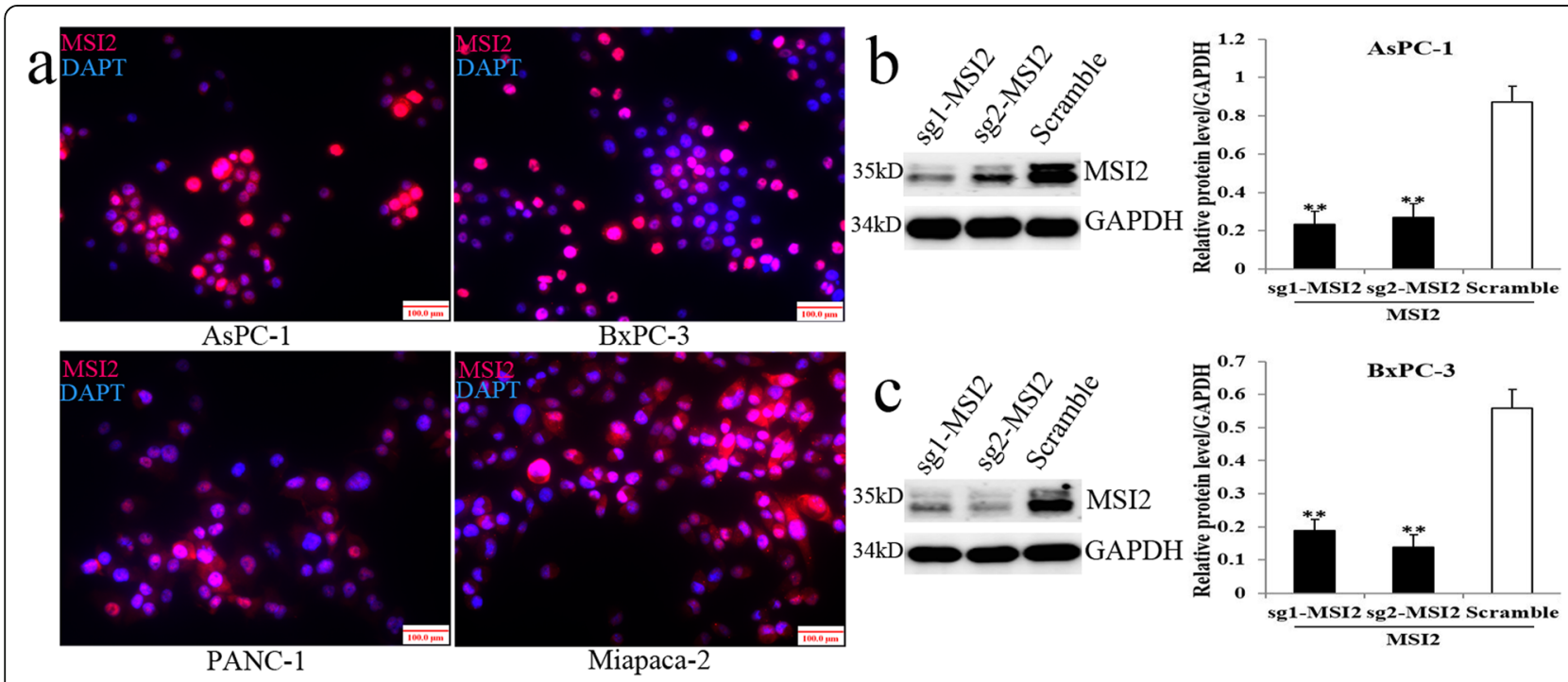

Fig. 1 Nuclear and cytoplasmic location of MSI2 in PC cells by IF and the construction of MSI2 silencing stable PC cell lines via CRISPR/Cas9 system. a IF staining of MSI2 (TRITC, red) and nuclear (Hoechst, blue) in 4 PC cell lines (× 100 magnification). b and c MSI2 protein level in sg1MSI2, sg2-MSI2 and scramble transfectedAsPC-1 (b) and BxPC-3 (c) cell lines detected by WB. The white bars: MSI2 protein expression in scramble groups. The black bars: MSI2 protein expression in sg1-MSI2 and sg2-MSI2 groups. **, $P<0.01$ compared with the control 

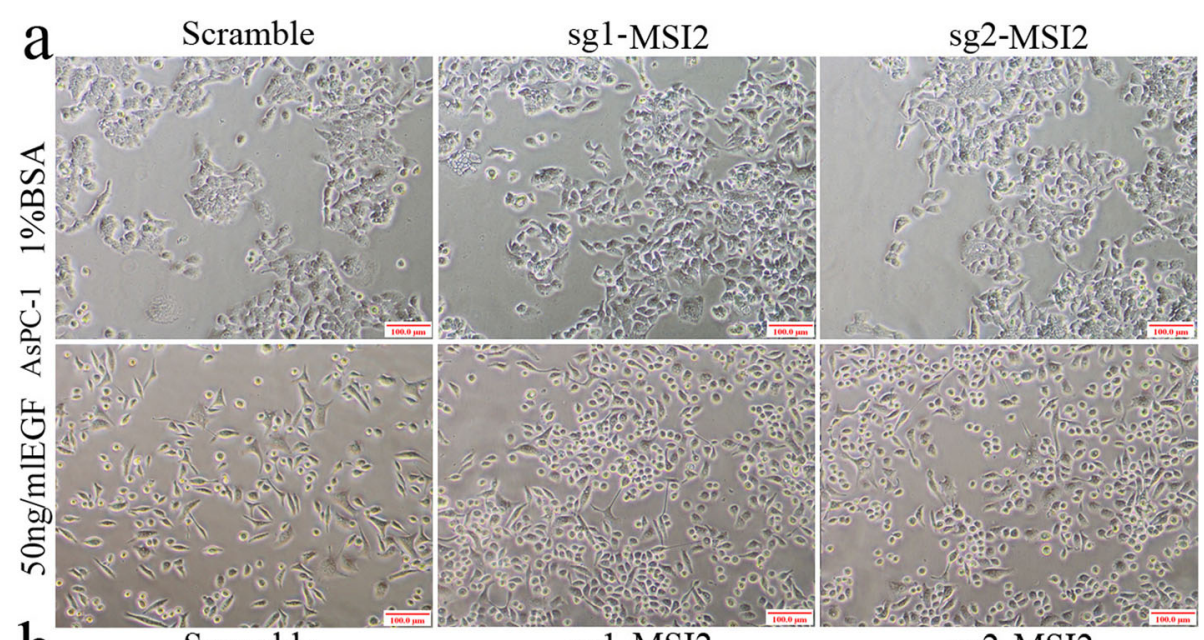

$\mathrm{b}$
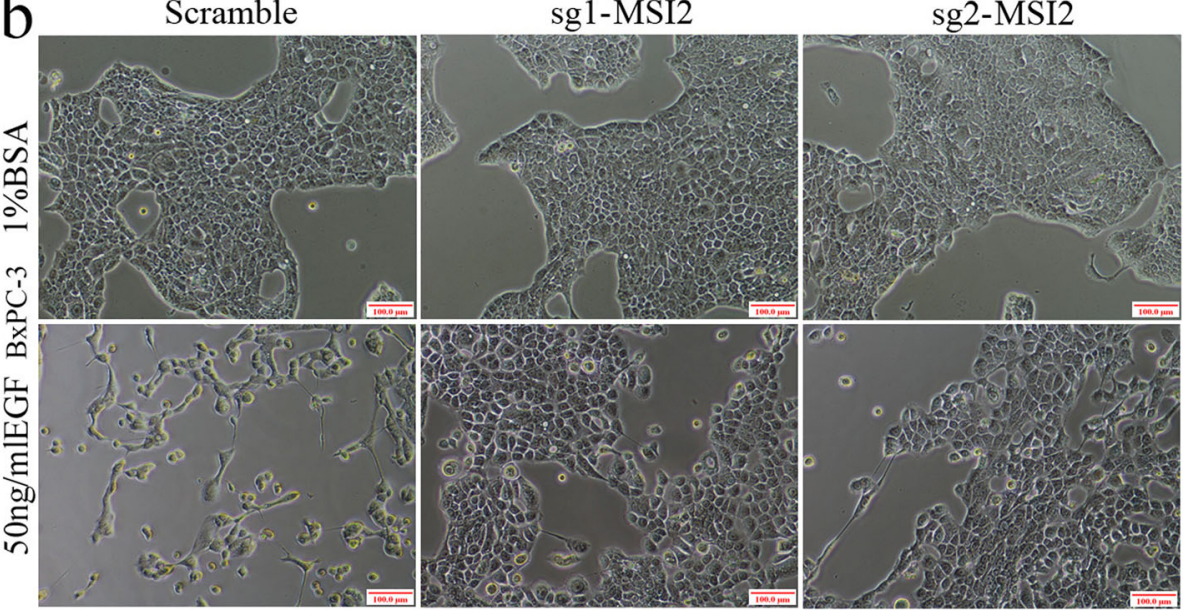

Fig. 2 Cell morphology in sg1-MSI2, sg2-MSI2 and scramble transfected AsPC-1 and BxPC-3 cells with or without EGF (50 ng/ml) treatment. a and b Under EGF treatment, the fibroblastoid-like phenotype in AsPC-1 (a) and BxPC-3 (b) cells in scramble, sg1-MSI2 and sg2-MSI2 groups

gels, transferred to PVDF membranes and incubated with primary antibodies: MSI2 (Abcam), ZEB1 (Proteintech), Ecad (Abcam), N-cadherin (N-cad, Abcam), c-Myc (Proteintech), pERK (Cell signaling technology), Cavelino-1 (Proteintech), ZO-1 (Proteintech), $\beta$-catenin (Proteintech), MMP9 (Abcam), Vimentin (Proteintech), alpha smooth muscle actin (a-SMA, Abcam), GAPDH (Proteintech), the phosphorylated epidermal growth factor receptor (EGFR) at tyrosine 845 (pEGFR845, Abcam), the phosphorylated site at tyrosine 992 (pEGFR992, Cell signaling technology) and at tyrosine 1068 (pEGFR1068, Abcam). Then, membranes were incubated with secondary antibodies (Proteintech). Protein bands were detected with an ECL detection kit (Thermol Biotech Inc., USA). Each experiment was repeated 3 times.

\section{Immunoprecipitation}

As described previously [18], whole protein lysates prepared from AsPC-1 and BxPC-3 cells were extracted in the IP lysis buffer. Briefly, MSI2, ZEB1, c-Myc and pERK and IgG (Santa Cruz) antibodies were pre-incubated with magnetic beads (Bio-Rad, California, USA) at $4{ }^{\circ} \mathrm{C}$. Then antibody-beads complex was washed 3 times and incubated with the supernatants of protein lysates overnight at $4{ }^{\circ} \mathrm{C}$. The immunocomplexes were eluted by boiling in sample loading buffer for SDS-PAGE, and then subjected to WB analysis with MSI2, ZEB1, pERK and c-Myc antibodies. Each experiment was repeated 3 times.

\section{CRISPR/Cas9 and siRNA mediated MSI2 silencing and lentivirus vector mediated MSI2 overexpression}

Lentivirus(Lenti)-cas9 and lenti-sgRNA were synthesized from Genechem (Genechem Co, Ltd., Shanghai, China). AsPC-1 and BxPC-3 cell lines were firstly transfected with lenti-cas 9 and next selected by puromycin (Sigma). The stable sub-lines were then transfected with MSI2-sgRNA (sg1-MSI2 and sg2-MSI2) and sgRNA control (scramble) to specifically silence target genes. MSI2siRNA and siRNAcontrol were synthesized from GenePharma Company 
(GenePharma Co, Ltd). Cells were transiently transfected with siRNA via oligofectamine3000 (Invitrogen, Carlsbad, CA, USA) under the protocol. Lentivirus vector (GV358) mediated MSI2 overexpression (MSI2-GFP) and corresponding control vector (GFP) were also synthesized from Genechem. SW1990 cells with MSI2 low expression was used to construct MSI2 overexpressing stable PC cells following puromycin selection. The vector information and stable transfected efficiency (GFP fluorescence in MSI2 overexpressing stable SW1990 cells) were shown in Additional file 1: Figure S1. All target sequences mentioned above were shown in Additional file 2: Table S1.

\section{EMT construction}

As our previous studies shown $[5,18]$, AsPC-1 and BxPC3 cell transfected with sg1-MSI2, sg2-MSI2 and scramble were treated with $50 \mathrm{ng} / \mathrm{ml}$ EGF (Peprotech, RockyHill, New Jersey, USA) and 1\%BSA (Sigma) triple times within $72 \mathrm{~h}$, respectively. Cells were cultured with recommended growth media containing 0.5\% FBS to enhance the efficiency of EGF. MSI-GFP and GFP transfected SW1990 cells were co-transfected with MSI2siRNA and treated with $50 \mathrm{ng} / \mathrm{ml}$ EGF mentioned above. The EMT construction was verified by the observation of EMT-like cell morphology (a spindle-shaped and fibroblast-like morphology), EMT-enhanced cell invasion and migration, and EMT-induced the change of EMT markers.

\section{Invasion and migration assays}

Cell invasion were assessed with modified Boyden chamber (BD Biosciences, Sparks, MD, USA) assays. Briefly, sg1-MSI2, sg2-MSI2 and scramble transfected PC cells (pretreated with EGF for $48 \mathrm{~h}$ ) was seeded onto 8.0- $\mu \mathrm{M}$ pore size membrane inserts coated with matrigel (BD Biosciences) in 24 well plates with FBS-free growth media plus EGF. Growth media plus 10\% FBS was added to the bottom wells as a chemoattractant. Twenty four hours later, cells that had migrated to the underside of the inserts were stained with Crystal Violet Hydrate (Sigma, St. Louis, MO, USA). The migratory cells were counted in five random fields per well. Each experiment was repeated three times.

\section{In vivo xenograft model}

Animals were maintained according to institutional regulations in facilities approved by the Animal Care Committee of China Medical University.

Total 20 nude mice $(B A L B / c-n u)$ were acclimatized for a week. Ten nude mice were used to construct pancreatic tumor in situ implanted with BxPC-3 cells (originated from primary $\mathrm{PC}$ ). The other 10 nude mice were used to construct liver metastasis model implanted with AsPC-1 cells (originated from ascites of PC).

For pancreatic tumor in situ, a small horizontal laparotomy incision $(1 \mathrm{~cm})$ was made in left abdominal flank under chloral hydrate anesthesia. The spleen was identified

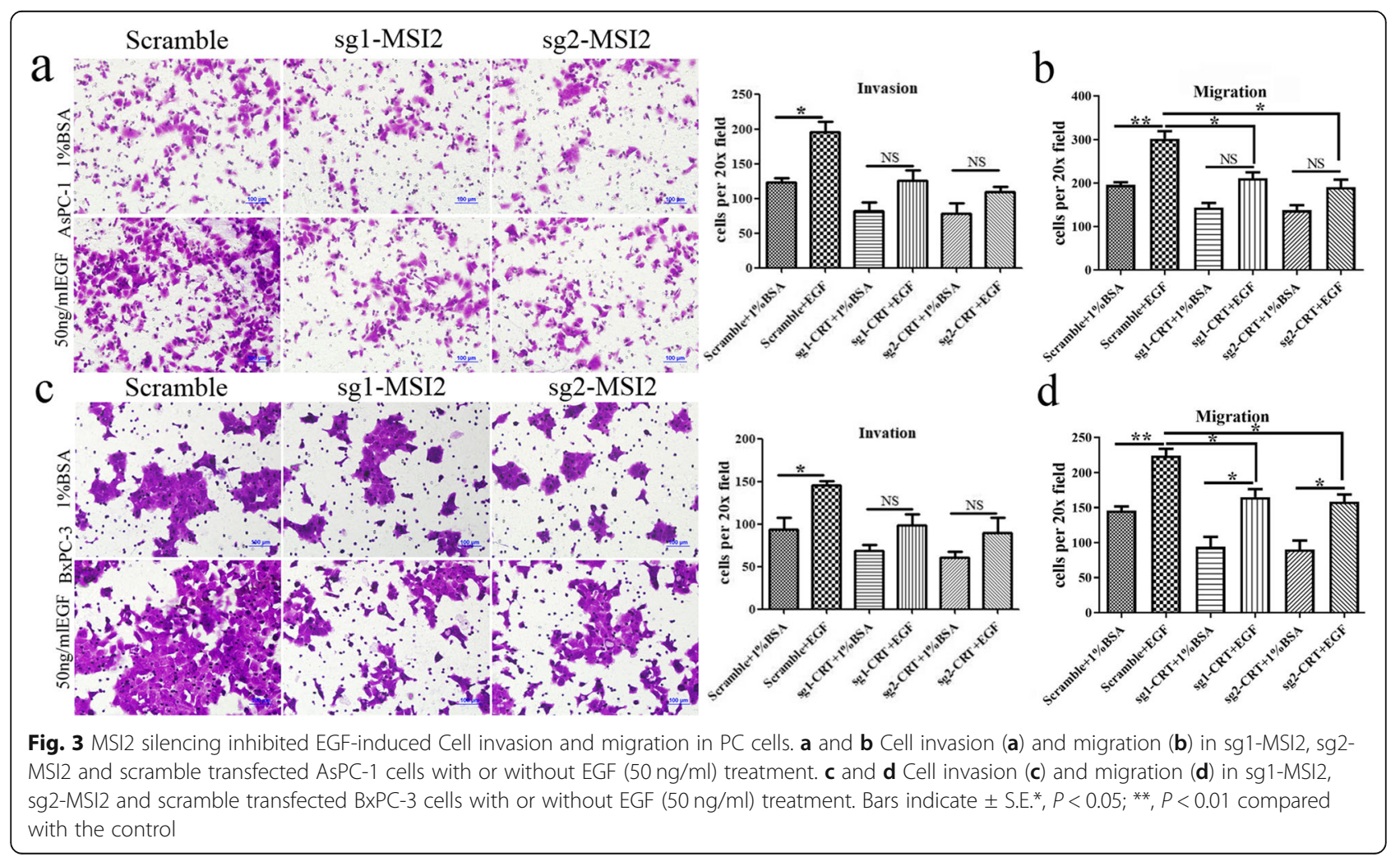




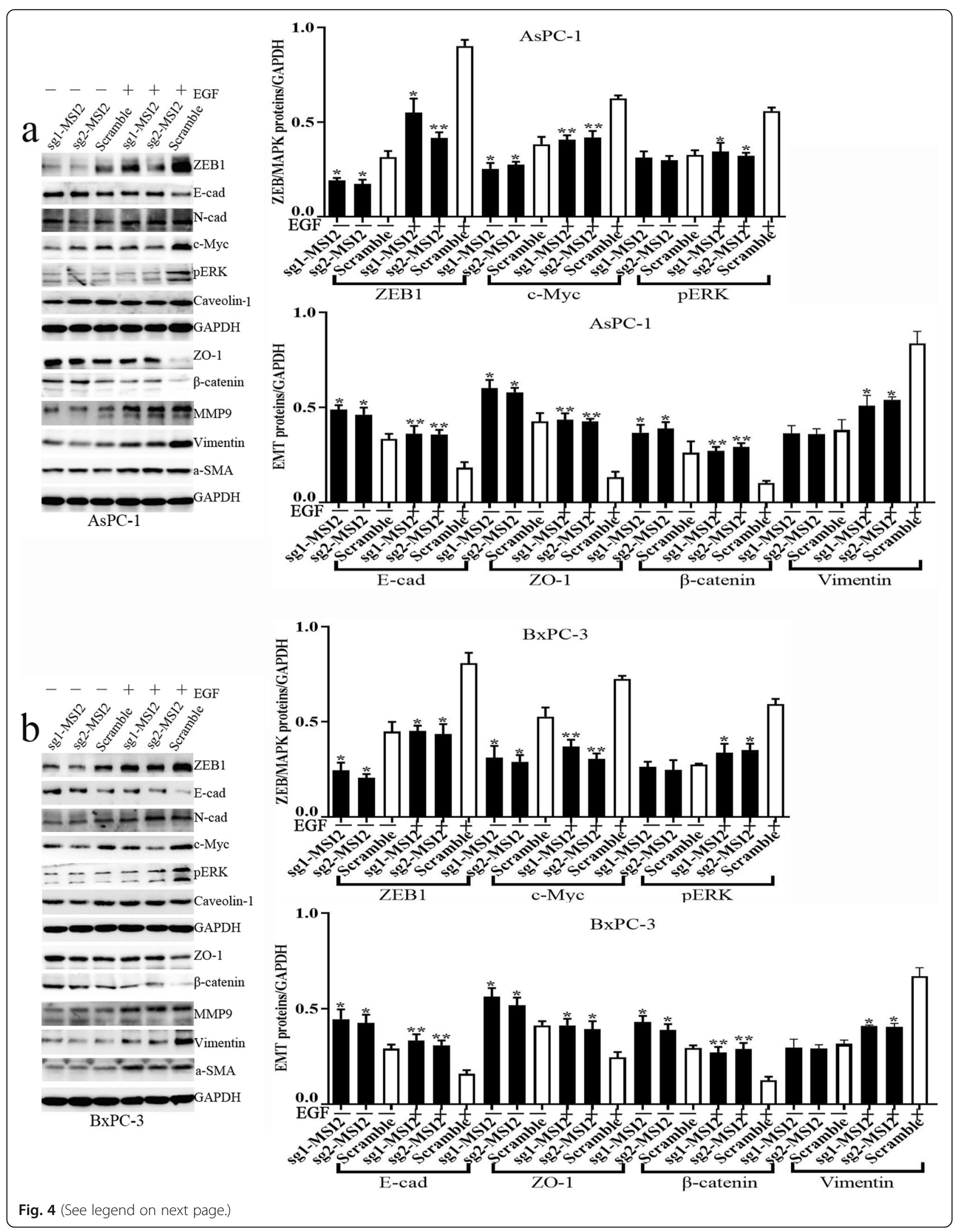


(See figure on previous page.)

Fig. 4 MSI2 silencing inhibited EGF-induced EMT and ZEB1-ERK/MAPK signaling. a and b. The protein expression involving EMT and ZEB1-ERKV MAPK signaling in sg1-MSI2, sg2-MSI2 and scramble transfected AsPC-1 (a) and BxPC-3 (b) cells with or without EGF (50 ng/ml) treatment. The white bars: target protein expression in scramble groups with or without EGF treatment. The black bars: target protein expression in sg1-MSI2 and sg2-MSI2 groups with or without EGF treatment. Bars indicate \pm S.E.* ${ }^{*}, P<0.05 ;{ }^{* *}, P<0.01$ compared with the control

and exposed. The injection site was the junction between spleen and pancreatic tail. sg-MSI2 and scramble transfected BxPC-3 cells $\left(2 \times 10^{7} / \mathrm{ml}\right)$ were suspended in precold PBS (50ul) mixed with Matrigel (50ul) (BD Biosciences) and were then transplanted into tail of pancreas in10 nude mice. A cotton swab was used to avoid possible bleeding and leakage. The injection operation was shown in Additional file 3: Video S1.

The mice were sacrificed 1 month later. Tumors were resected and calculated using vernier calipers with the following formula: length $\mathrm{x}$ width $\mathrm{x}$ height $\times 0.52$ in millimeters. Then, samples were extracted for late hematoxylin and eosin (HE) and IHC staining.

For liver metastasis model, as described in our previous studies [19], using the same incision mentioned above, the spleen was identified and exposed. A total of $1 \times 10^{\wedge} 7 / \mathrm{ml}$ sg-MSI2 and scramble transfected AsPC-1 cells suspended in pre-cold PBS (200ul) were injected into the lowermiddle part of splenic capsule. A cotton swab was used to avoid bleeding and leakage from the injection site. The injection operation was shown in Additional file 4: Video S2. The mice were sacrificed 3 weeks later. The number of

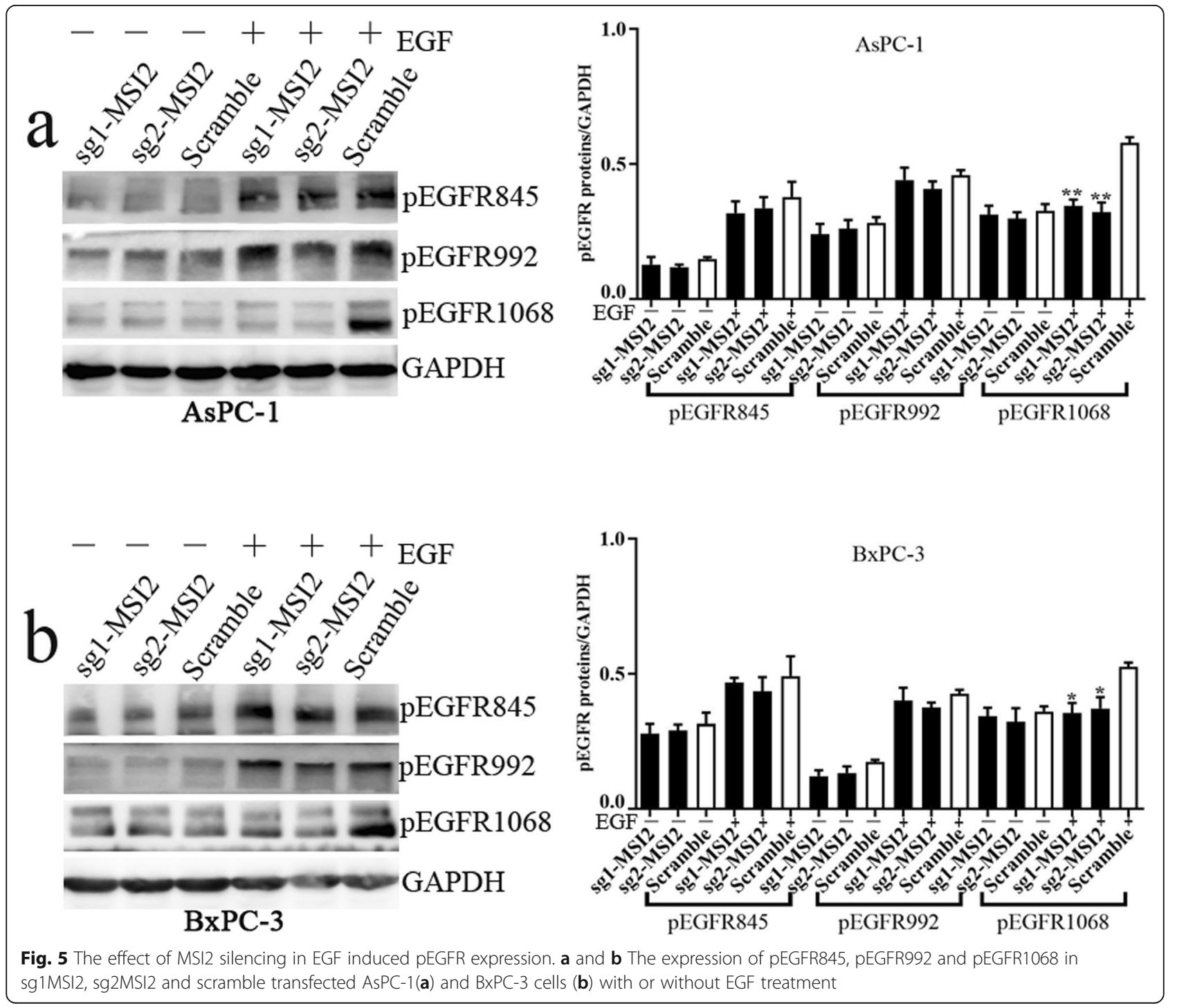



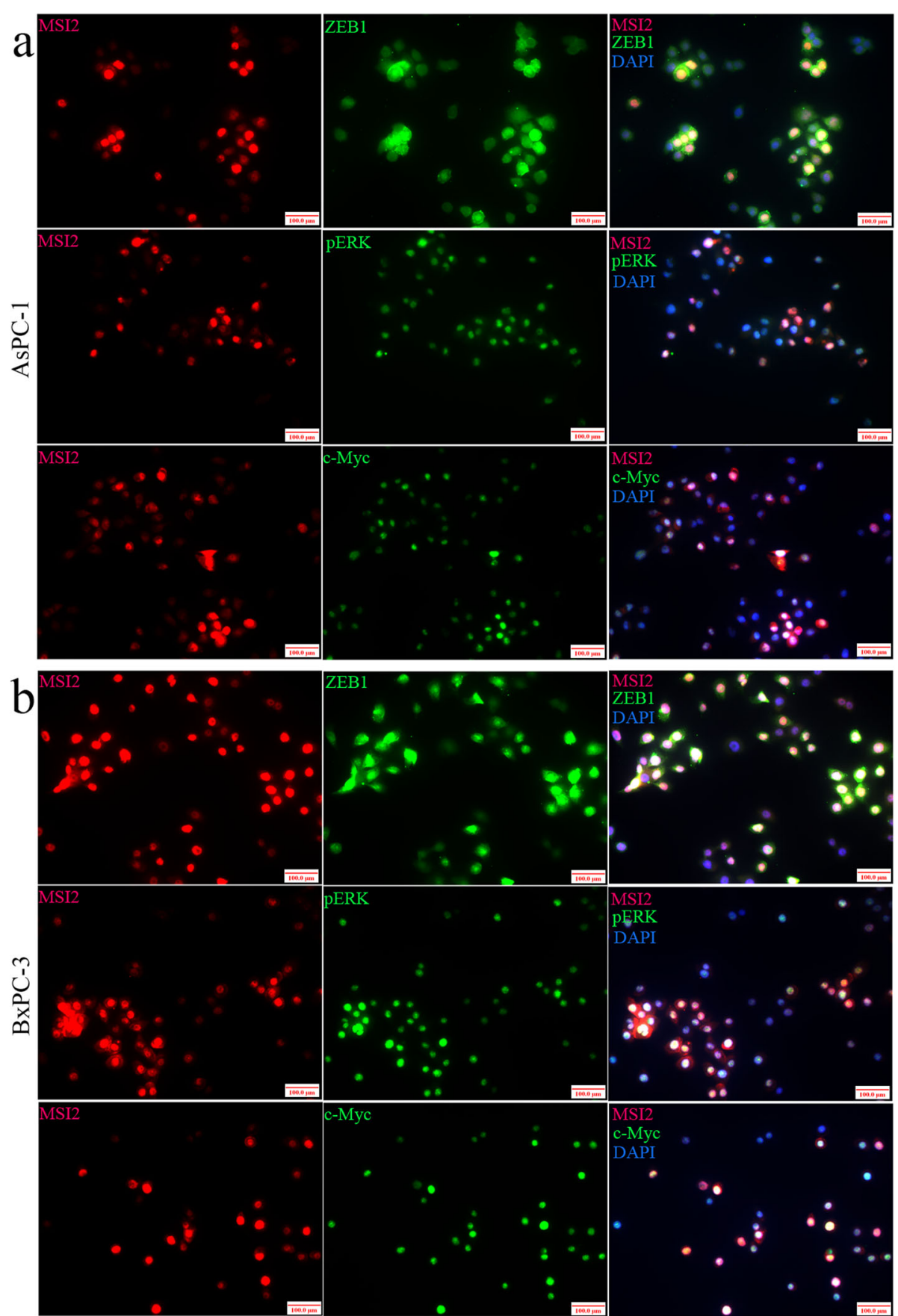

Fig. 6 The co-staining of MSI2 with ZBE1, pERK and c-Myc in PC cells by IF. a and $\mathbf{b}$ MSI2 was co-stained with ZBE1, pERK and c-Myc in predominant nucleus in AsPC-1 (a) and BxPC-3 (b) cells ( $\times 100$ magnification)

liver metastases was investigated immediately, and fixed for late HE and IHC staining.

\section{Statistical analysis}

Using SPSS software 20.0 (SPSS, Chicago, IL, USA). The differences in WB, transwell assays, the volumes in orthotopic tumor and the numbers in liver metastases were expressed as mean $\pm S E$ and compared through Student's $t$-test. The different proteins expression in vivo by IHC were compared through nonparametric test. The relationship between each target protein in human PC samples were analyzed by Spearman correlation tests. The Kaplan-Meier curve was used to estimate survival, and differences were analyzed by the log-rank test. A value of $P<0.05$ indicated statistic significant. 


\section{Results}

MSI2 location and the construction of MSI2 silencing stable cell lines

MSI2 showed predominantly nuclear and cytoplasmic expression in AsPC-1, BxPC-3, PANC-1 and Miapaca-2 cell lines by IF (Fig. 1a). Our previous studies showed EGF successfully induced EMT in MSI2 high expression AsPC-1 and BxPC-3 cell lines [5, 18]. Thus, CRISPR/ Cas9 system mediated MSI2 silencing was constructed in above cell lines for the late EMT study. WB showed that MSI2 protein level in both 2 PC cell lines in sg1MSI2 and sg2-MSI2 groups were significantly lower than that in corresponding scramble groups (Fig. 1b and c).

\section{MSI2 silencing inhibited EGF-induced EMT in 2 PC cell} lines

Upon EGF, both AsPC-1 and BxPC-3 cells exhibited EMT-like cell morphology: cells lost their epithelial character, and exhibited a spindle-shaped and fibroblastlike morphology (Fig. 2). MSI2 silencing alone had no effect in cell morphology, but significantly inhibited EGFinduced EMT-like cell morphology. In sg1-MSI2 and sg2-MSI2 groups, EGF-treated AsPC-1 and BxPC-3 cells restored their original cell morphology with little spindle-shaped and fibroblast-like morphology, compared with scramble groups (Fig. 2).

The aggressiveness of PC cells is significantly driven by EMT [4]. We found that EGF significantly stimulated cell invasion and migration in both AsPC-1 and BxPC-3 cells (Fig. 3). MSI2 silencing alone without EGF partially inhibited cell invasion and migration. However, this trend is much more significant with EGF treatment. Upon EGF, a significant increase of cell invasion and migration were found in scramble groups compared with sg1-MSI2 and sg2-MSI2 groups. Namely, the growing difference of cell motility between scramble groups with and without EGF was much more obvious than that in sg-MSI2 groups (Fig. 3).

Meanwhile, EGF significantly induced the change of EMT epithelial and mesenchyme markers in PC cells. In detail, EGF induced decrease of epithelial markers E-cad, $\mathrm{ZO}-1$ and $\beta$-catenin and increase of mesenchyme markers MMP9, vimentin and a-SMA in both AsPC-1 and BxPC-3 cells (Fig. 4). MSI2 silence alone without EGF stimulus partially upregulated E-cad, ZO-1 and $\beta$ catenin expression. This trend is much more significant

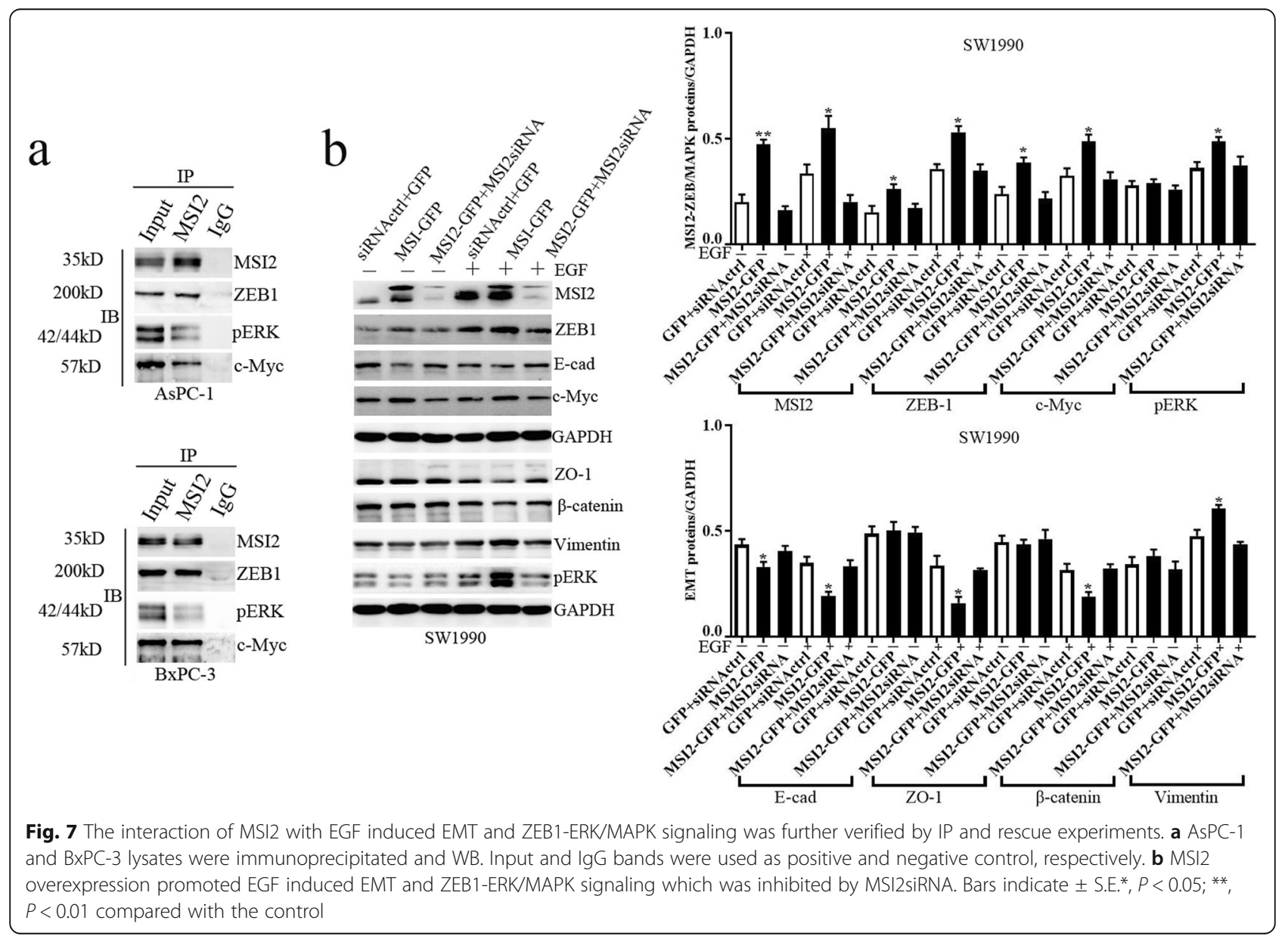



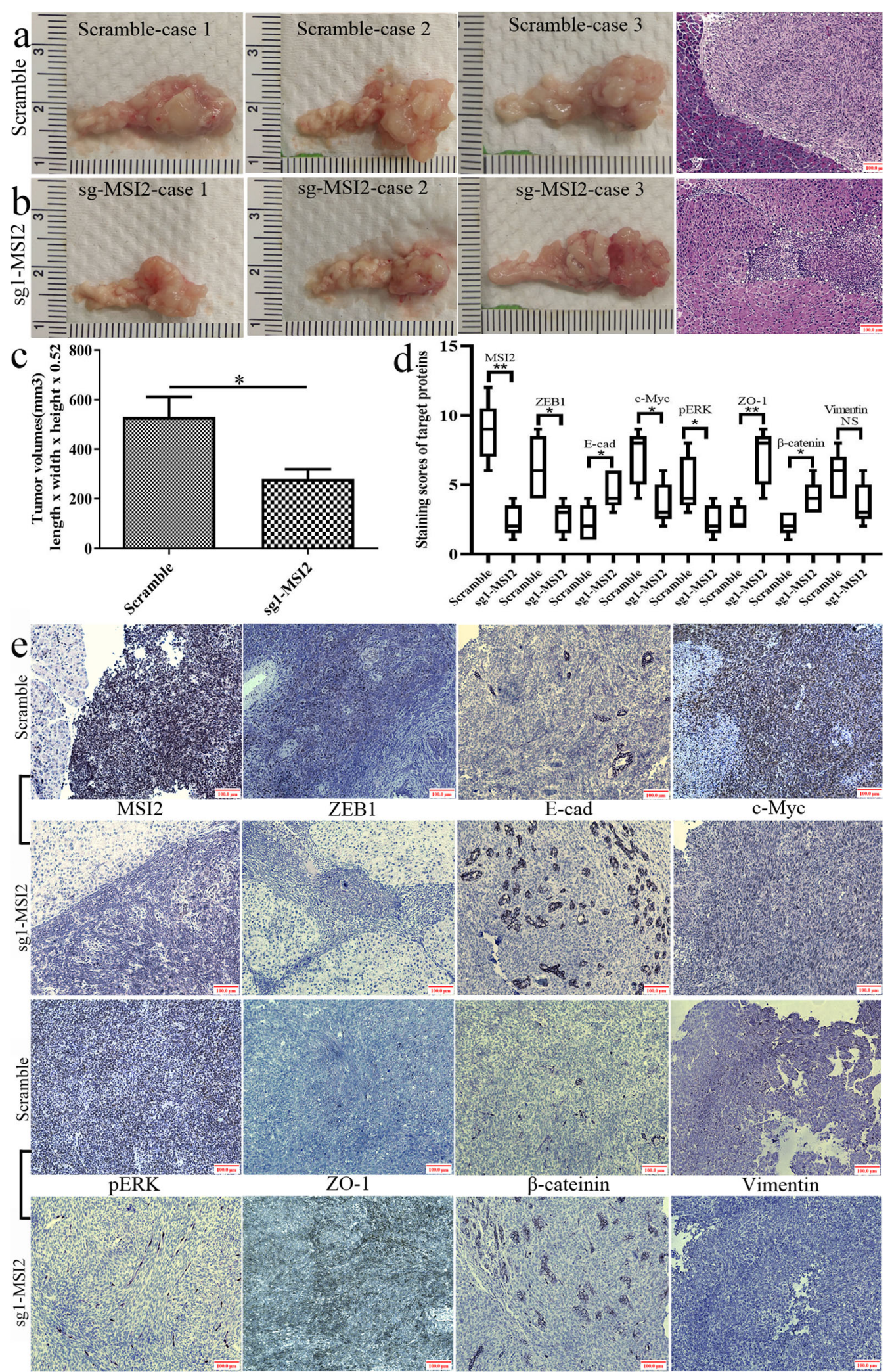

Fig. 8 (See legend on next page.) 
(See figure on previous page.)

Fig. 8 MSI2 silencing inhibited the growth of pancreatic tumors in situ. a Pancreatic tumors and corresponding HE staining $(\times 100$ magnification $)$ in scramble groups. b Pancreatic tumors and corresponding HE staining ( $\times 100$ magnification) in sg1-MSI2 groups. c The statistic analysis of tumor volume between scramble and sg1-MSI2 groups. $\mathbf{d}$ and $\mathbf{e}$ The statistic data (d) and representative images (e) in IHC assays of target proteins expression in vivo in sg1-MSI2 and scramble groups. Bars indicate \pm S.E.*, $P<0.05 ;{ }^{* *}, P<0.01$ compared with the control

upon EGF. Moreover, MSI2 silencing also inhibit EGFinduced increase of vimentin (Fig. 4).

Taken together, MSI2 silencing inhibited EGF induced EMT in vitro.

\section{MSI2 silencing inhibited EGF-stimulated ZEB1-ERK/MAPK signaling in 2 PC cells}

It is well known that EGF is the prototypic and founding member of the EGFR ligand family. EGF binding triggers receptor autophosphorylation on key cytoplasmic residues. The phosphorylated receptor activates complex downstream signaling cascades, including the ERK/MAPK pathway. Thus, we first detected the expression of phosphorylated EGFR at different sites of tyrosine in EGF treated PC cells upon EGF. In Fig. 5, EGF activated phosphorylation of EGFR at the sites of tyrosine 845 (pEGFR845), 992 (pEGFR992) and 1068 (pEGFR1068) in vitro. All their expression was significantly increased in EGF treated AsPC-1 and BxPC-3 cells. However, MSI2 silence specifically inhibited EGF activated pEGFR1068 in both cell lines. The expression of pEGFR845 and pEGFR992 were unchanged (Fig. 5). Thus, we inferred that MSI2, as an upstream regulator, specially regulated EGF activated pEGFR1068 and its downstream of ERK/ MAPK signaling in PC cells.

ZEB1 is a key element of a network of transcription factors that controls EMT [20]. ERK/MAPK pathway and its key downstream target c-Myc plays a vital role in the initiation of EMT in normal epithelial and cancer cells [21-25]. Meanwhile, EMT in lung cancer cells was meditated by the close interaction between ERK and ZEB1 pathway [26]. Thus, we next investigated whether MSI2 mediated EGF induced EMT via ZEB1-ERK/ MAPK signaling.

In current study, EGF stimulated ZEB1-ERK/MAPK signaling in both AsPC-1 and BxPC-3 cells along with the increase of ZEB1, pERK and c-Myc protein expression (Fig. 4). Without EGF, MSI2 silence alone partially inhibited ZEB1 and c-Myc expression. Upon EGF, a significant decrease of ZEB1, pERK and c-Myc were observed in sgMSI2 and sgMSI2 groups compared with the corresponding scramble groups (Fig. 4). Meanwhile, MSI2/ZEB1, MSI2/pERK and MSI2/c-Myc were costained in predominant nuclear in normal AsPC-1 and BxPC-3 cells by IF (Fig. 6). Moreover, MSI2 was coimmunoprecipitated with above proteins in the lysates in both cell lines (Fig. 7a), implying a close interaction between MSI2 and ZEB1-ERK/MAPK signaling pathway.
For the rescue experiment, SW1990 PC cells with low MSI2 expression were firstly used to construct MSI2 overexpression stable cell line (MSI2-GFP). WB showed that MSI2 expression was significantly higher in MSI2GFP group than that in scramble GFP group whatever with or without EGF treatment (Fig. 7b). Meanwhile, MSI2 overexpression in MSI2-GFP group was significantly inhibited when co-transfected with MSI2siRNA (Fig. 7b). Without EGF, MSI2 overexpression upregulated ZEB1 and c-Myc and downregulated E-cad expression, which was rescued by MSI2siRNA. Upon EGF, MSI2 overexpression promoted EGF-induced decrease of E-cad, ZO-1 and $\beta$-catenin and enhanced EGFinduced increase of c-Myc, pERK and Vimentin, all which were reversed by MSI2siRNA. Taken together with CRISPR/Cas9 mediated MSI2 silencing experiments, we conclude that MSI2 has a specific regulation in EGF-induced EMT in PC cells via ZEB1-ERK/MAPK signaling.

\section{MSI2 silencing inhibited pancreatic tumors in situ and liver metastasis in vivo}

BxPC-3 and AsPC-1 cells were used to construct the models of pancreatic tumor in situ and distal liver metastasis, respectively.

Tumor volumes in the pancreas in sg1-MSI2 group were much smaller than that in paired corresponding scramble groups $(P=0.031)$ (Fig. $8 \mathrm{a}-\mathrm{c})$. HE staining also showed a large tumor area in scramble group compared with that in sg1-MSI2 groups (Fig. 8a, b). IHC further verified that MSI2, ZEB1, c-Myc and pERK expression were decreased (Fig. 8d, e) but E-cad, ZO-1 and $\beta$ catenin were increased in sg1-MSI2 groups compared with that in scramble groups (Fig. 8d, e). Vimentin showed high expression in scramble group, but no significant difference with sg1-MSI2 group.

The liver metastases in sg1-MSI2 group was much lower than that in scramble groups $(P=0.009)$ (Fig. 9ac). HE staining also showed a large and serial area of liver metastasis in scramble group compared with that in sg-MSI2 groups only with scattered dot diffusion (Fig. 9a, b). IHC further verified that MSI2, ZEB1, c-Myc and pERK expression were increased, but E-cad, ZO-1 and $\beta$-catenin expression were decreased in sg1-MSI2 compared with the scramble groups (Fig. 9d, e). Vimentin showed high expression in scramble group, but no significant difference with sg1-MSI2 group. 


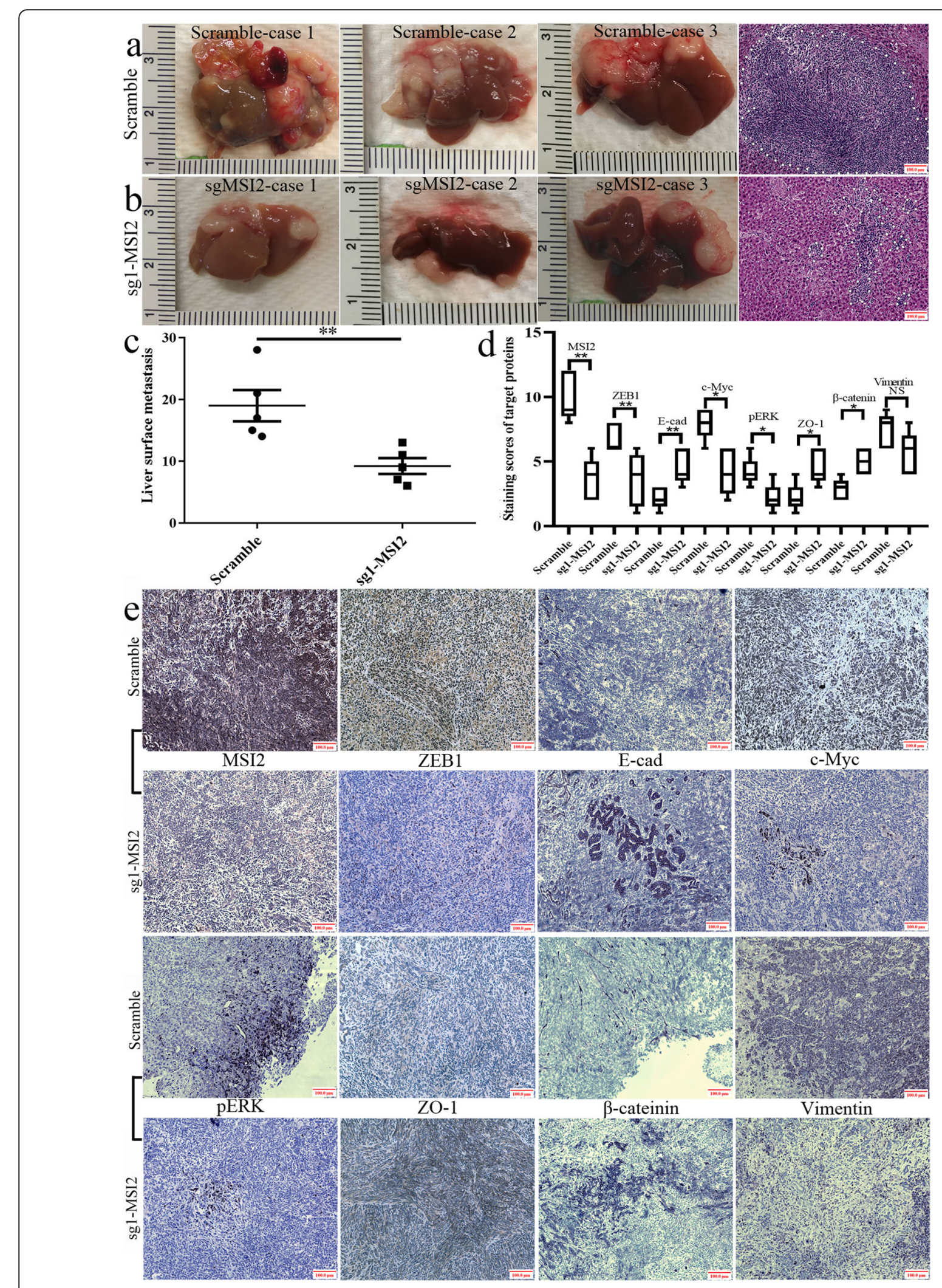

Fig. 9 (See legend on next page.) 
(See figure on previous page.)

Fig. 9 MSI2 silencing inhibited liver metastases in vivo. a Liver metastases and corresponding HE staining ( $\times 100$ magnification) in scramble groups. b Liver metastases and corresponding HE staining ( $\times 100$ magnification) in sg1-MSI2 groups. c The statistic analysis of liver metastasis number between scramble and sg1-MSI2 groups. $\mathbf{d}$ and $\mathbf{e}$ The statistic data (d) and representative images (e) in IHC assays of target proteins expression in vivo in sg1-MSI2 and scramble groups. Bars indicate \pm S.E. ${ }^{*}, P<0.05$; ${ }^{* *}, P<0.01$ compared with the control

\section{Clinical significance and the relationship of MSI2 with} EMT and ZEB1-ERK/MAPK signaling in human PC samples Finally, we investigated the close relationship between MSI2, ZEB1 (the key regulator of EMT), c-Myc (the key target of ERK/MAPK signaling), E-cad (EMT epithelial marker) and vimentin (EMT mesenchyme markers) with the clinical significance of PC patients.

The location of MSI2, ZEB1 and c-Myc in nucleus sand cytoplasm, and vimentin in membrane and cytoplasm were considered for scoring. E-cad membrane expression was identified as normal expression, whereas its negative or cytoplasmic expression were identified as abnormal expression. In 74 PC samples, proteins were overexpressed in $67.6 \%(50 / 74)$ of MSI2, 52.7\% (39/74) of ZEB1, 39.2\% (29/74) of E-cad, 51.3\% (38/74) of c-Myc and $29.7 \%(22 / 74)$ of vimentin by IHC assays, respectively (Table 1 ).

MSI2 was positively associated with ZEB1 and c-Myc expression $(P=0.021$ and $P=0.032)$ and negatively associated with normal E-cad expression $(P=0.019)$, but had no relationship with vimentin. In serial sections, PC tissues with high MSI2 expression were associated with positive ZEB1, c-Myc and abnormal E-cad expression (Fig. 10a), and vice versa (Fig. 10b). No association was found between MSI2 and vimentin, though their coexpression was showed in most PC tissues. These results were consistent with our observation in vitro and vivo.

The clinical significance of above protein in PC patients are well studied in our previous study and other reports [16, 17, 27-29]. Briefly, abnormal expression of above proteins was associated with multiple advanced clinical characters in PC patients. In current study, we concentrated on the relationship of their coordinate

Table 1 The relationship of MSI2 with ZEB1, E-cad, Vimentin and c-Myc expression in clinical samples

\begin{tabular}{llllll}
\hline Parameters & CRT & \multicolumn{2}{l}{$\begin{array}{l}\text { Spearman } \\
\text { ranks }\end{array}$} & $P$ \\
\cline { 3 - 4 } & & Low (24) & High (50) & & \\
\hline ZEB1 & Negative (35) & 16 & 19 & 0.269 & 0.021 \\
& Positive (39) & 8 & 31 & & \\
E-cad & Abnormal (45) & 10 & 35 & -0.272 & 0.019 \\
& Normal (29) & 14 & 15 & & \\
c-Myc & Negative (36) & 16 & 20 & 0.250 & 0.032 \\
& Positive (38) & 8 & 30 & & \\
Vimentin & Negative (52) & 20 & 32 & 0.198 & 0.089 \\
& Positive (22) & 4 & 18 & & \\
\hline
\end{tabular}

expression with the prognosis of PC patients. High expression of MSI2 and ZEB1 were associated with the poor prognosis of $\mathrm{PC}$ patients, respectively $(P=0.007$ and $P=0.021$ ) (Fig. 11a, b). Moreover, patients with the co-expression of MSI2 and ZEB1 had a much worse prognosis than patients with their low expression $(P=$ 0.003) (Fig. 11e). Though E-cad and c-Myc had no relationship with the survival (Fig. 10c, d), patients combining with high MSI2 and abnormal E-cad expression or high MSI2 and c-Myc expression were associated with the worse prognosis $(P=0.013$ and $P=0.020)$ (Fig. 10f, g). Taken together, co-expression of above proteins coordinately promoted the aggressive stage and poor prognosis of PC patients.

\section{Discussion}

Accumulating studies have elucidated the frequency and mechanisms of MSI2 in multiple human hematology and solid cancers. For example, MSI2 silencing in AML cells increases sensitivity toward daunorubicin treatment via downregulating BCL2 and upregulating BAX [30]. MSI2 overexpression in a murine model of CML leads to downregulation of Numb by binding to the mRNA and preventing its translation [11]. MSI2 promotes the stemness and chemoresistance in liver cancer stem cells via LIN28A activation [31]. MSI2 promotes cell growth in breast cancer via increasing estrogen receptor 1 protein stability [32]. Our previous study also showed MSI2 promoted cell invasion and metastasis of PC by downregulating Numb and enhanced drug resistance in p53dependent manner $[15,16]$. In current study, MSI2 promoted EGF-induced EMT in PC via ZEB1-ERK/MAPK signaling in vitro and vivo, which is a novel signaling in oncogenesis, to our knowledge.

We first found MSI2 silencing inhibited EGF-induced EMT in 2 PC cells, including inhibiting EGF-induced EMT-like cell morphology, EGF-enhanced cell invasion and migration, and EGF-stimulated change of EMT epithelia and mesenchyme markers. Similarly, MSI2 silencing represses extrahepatic cholangiocarcinoma growth and invasion by inhibiting EMT [33]. MSI2 silencing impairs cell proliferation and EMT in esophageal squamous cell carcinoma [34]. MSI2 depletion significantly downregulates EMT markers (TGF- $\beta$ receptor 1 , SMAD3, SNAI1 and SNAI2) to inhibit non-small cell lung cancer metastasis [35]. Our study offers a new view involving the close interaction of MSI2 with EGF-induced EMT in $\mathrm{PC}$, which has not been reported to our knowledge. 


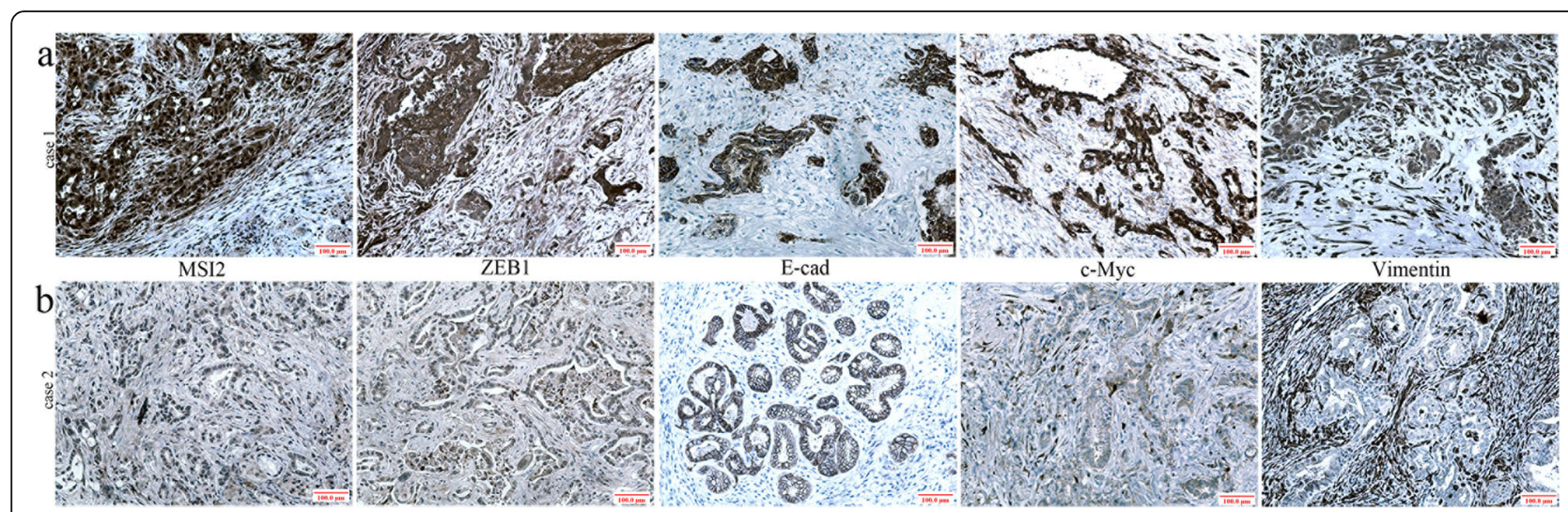

Fig. 10 MSI2, ZEB1, c-Myc, pERK, E-cad and Vimentin expression in human PC samples. a High expression of MSI2, ZEB1,c-Myc and Vimentin and abnormal expression of E-cad in one case of PC tissues. b Weak expression of MSI2, ZEB1, C-Myc and Vimentin and normal expression of E-cad in another one case of PC tissues

It is well known that EGFR plays a significant role in a number of varied processes in cancer progression such as cell adhesion, cell motility and invasion which are major steps in EMT event. Thus, we first detected if MSI2 regulated EGF induced EGFR phosphorylation in EMT inducing progression. We first found that MSI2, as an upstream regulator, specially regulated EGF activated pEGFR1068 and its downstream of ERK/MAPK signaling in PC cells.

ZEB1, as a transcription factor, promotes tumor invasion and metastasis by inducing EMT in many cancers [36-39]. ZEB1 recruiting multiple chromatin enzymes of
E-cad promoter, which is the key mechanism in regulating EMT [40]. ERK/MAPK pathway and its major target downstream c-Myc were also indispensable in EMT development in various mammary epithelial and cancer cells [21-25, 41, 42]. Both c-Myc and MEK1-induced ERK2 nucleus localization are required for TGF- $\beta$-induced EMT in prostate cancer [25]. Additionally, the ERK-ZEB1 pathway mediates EMT in pemetrexed resistant lung cancer cells [26]. Loss of E-cad stimulates EGFR-MEK/ERK signaling, which promotes invasion via the ZEB1/MMP2 axis in non-small cell lung cancer [43]. Based on above studies, we further investigated whether MSI2 mediated EGF

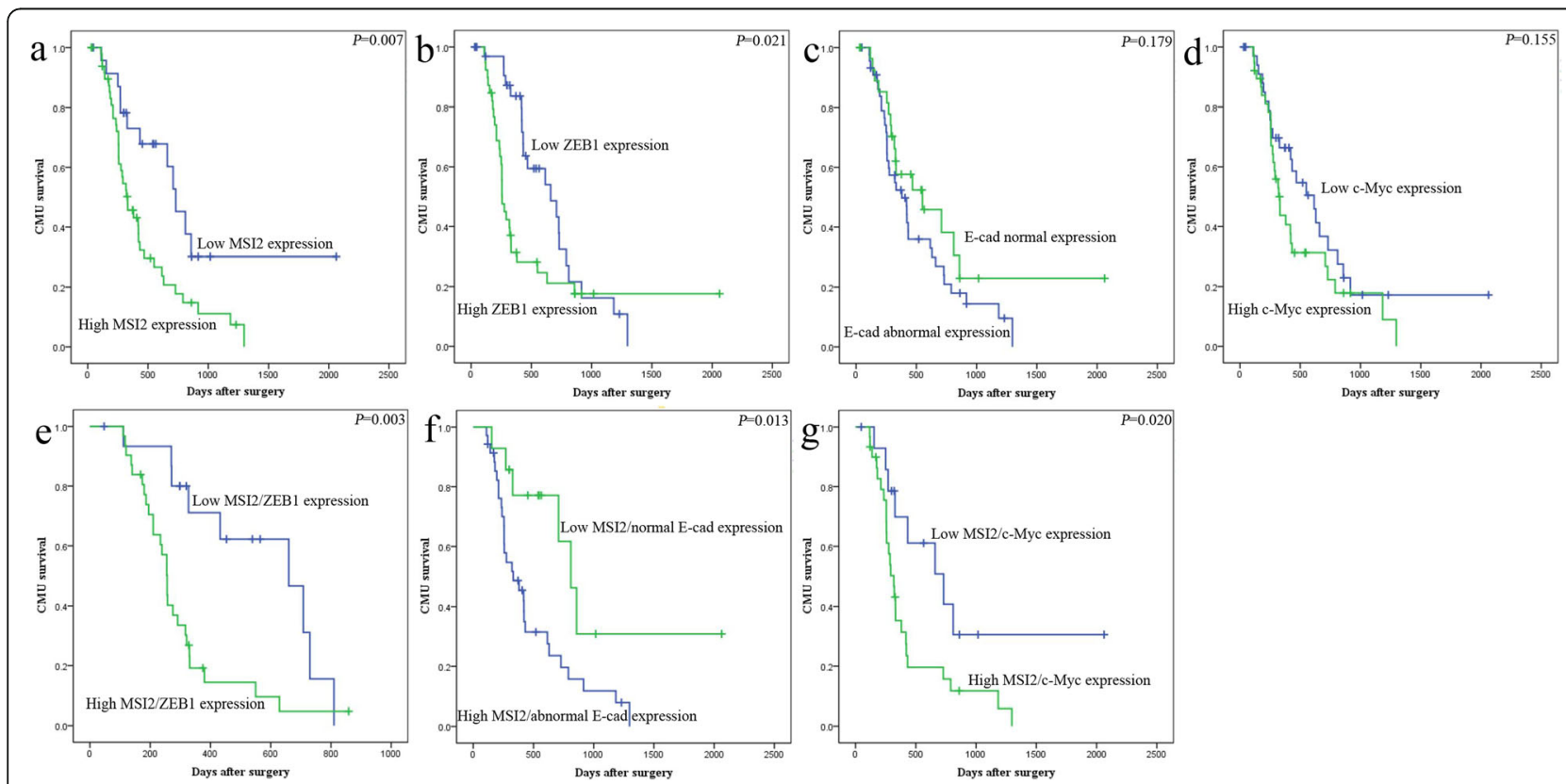

Fig. 11 The relationship of MSI2, ZEB1, c-Myc, pERK and E-cad with the survival of 74 postoperative PC patients in Kaplan-Meier analysis. a High and low expression of MSI2 was plotted against overall survival time. $\mathbf{b}$ High and low expression of ZEB1 was plotted against overall survival time. c Normal and abnormal expression of E-cad was plotted against overall survival time. $\mathbf{d}$ High and low expression of c-Myc was plotted against overall survival time. e, f and $\mathbf{g}$ Co-expression of MSI2/ZEB1 (e), MSI2/E-cad (f) and MSI2/C-Myc (g) was plotted against overall survival time 
induced EMT via ZEB1-ERK/MAPK signaling. In current study, EGF significantly activated ZEB1-ERK/MAPK signaling following with the increase of ZEB1, pERK and cMyc protein expression, which was significantly inhibited by MSI2 silencing. Meanwhile, MSI2 was co-stained and co-immunoprecipitated with ZBE1, pERK and c-Myc by IF and co-IP in both 2 PC cells. Moreover, further rescue experiments were verified above results. MSI2 overexpression promoted EGF induced EMT and ZEB1-ERK/MAPK signaling in vitro that was significantly inhibited by MSI2siRNA. In summary, with EGF stimulus, MSI2 interacted with ZEB1, upregulated ZEB1 and subsequently downregulated E-cad protein expression. Meanwhile, MSI2 promoted EGF-induced EGFR phosphorylation and EGFactivated ERK/MAPK signaling via interacted with the downstream target c-Myc. Finally, both above signaling pathways coordinately promoted EGF-induced EMT in PC cells mentioned above.

In vivo, MSI2 silencing inhibited pancreatic tumors in situ and liver metastasis, which was consistent with the studies in lung and esophageal squamous cell carcinoma $[34,44]$. Moreover, in parallel with the results in vitro, the close relationship between MSI2 with EMT and ZEB1-ERK/MAPK signaling was also observed in vivo and in human PC samples. Meanwhile, co-expression of above target proteins was closely associated with the poor prognosis of PC patients. Taken together, MSI2 and ZEB1-ERK/MAPK signaling cooperatively contributed to the advance development of PC.

\section{Conclusion}

In conclusion, MSI2 promotes EGF-induced EMT in PC cells via ZEB1-ERK/MAPK signaling pathway. Recently, accumulating studies focused on the function of the alternately spliced isoforms in MSI protein family. Upregulation of MSI2 variant 2 was observed in a large number of cancers by TCGA RNA-seq data sets [45]. Meanwhile, the small-molecule targeting of MSI2 activity is used in murine AML leukemia model [46]. The potential role of MSI2 isoforms and their small-molecule targets in PC will be further investigated in our future study.

\section{Supplementary information}

Supplementary information accompanies this paper at https://doi.org/10. 1186/s13046-020-1521-4

Additional file 1: Figure S1 The GV358 lentivirus vector information (a) and transfected efficiency (GFP fluorescence) in MSI2 overexpressing SW1990 cells (b).

Additional file 2: Table S1. The target sequences of sg1-MSI2, sg2-CRT, MSI2siRNA and corresponding scramble.

Additional file 3. The video of pancreas injection operation.

Additional file 4. The video of spleen injection operation.

\section{Abbreviations}

AML: Acute chronic myeloid leukemia; CML: Chronic myeloid leukemia; EGF: Epidermal growth factor; EGFR: Epidermal growth factor receptor; EMT: Epithelial mesenchymal transition; HE: Hematoxylin and eosin staining; IF: Immunofluorescence; IHC: Immunohistochemistry;

IP: Immunoprecipitation; MSI2: Musashi-2; PC: Pancreatic cancer; WB: Western Blot

\section{Acknowledgements}

We thank for the General Laboratory and the Center Laboratory of the First Hospital of China Medical University for technical supports.

\section{Authors' contributions}

Conception and design: WS, MD. Development of methodology: WS, SY, LY, TJ, CJ. Acquisition of data: LY, TJ, LY, WG, CR. Analysis and interpretation of data (statistical analysis, biostatistics, computational analysis): SY, LY, TJ, WG, SJ. Writing, review and/or revision of manuscript: WS, MD, WG.

Administrative, technical or material support: SY, LY, TJ, LY, CJ, CR, SJ. Study supervision: WS, MD. All authors read and approved the final manuscript.

\section{Funding}

This work was supported by Chinese National Science Foundation (NO. 81672835 to Ming Dong).

\section{Availability of data and materials}

Materials are available upon request.

\section{Ethics approval and consent to participate}

This research protocol was approved by the ethical committee of the institutional review board of China Medical University and a consent form was signed by each participating patient.

\section{Consent for publication}

Not applicable.

\section{Competing interests}

The authors declare that they have no competing interest.

\section{Author details}

${ }^{1}$ Department of Gastrointestinal Surgery, the First Hospital, China Medical University, Shenyang 110001, China. ${ }^{2}$ Department of General Surgery, the People's Hospital of Liaoning province, Shenyang 110034, China. ${ }^{3}$ Department of General Surgery, the First Hospital of Nanchang University, NanChang 330006, China. ${ }^{4}$ Department of General Surgery, the Central Hospital of JingZhou City, JingZhou 434020, China.

Received: 16 September 2019 Accepted: 6 January 2020

Published online: 17 January 2020

\section{References}

1. Chen W, Zheng R, Baade PD, Zhang S, Zeng H, Bray F, Jemal A, Yu XQ, He J. Cancer statistics in China, 2015. CA Cancer J Clin. 2016;66(2):115-32.

2. Siegel RL, Miller KD, Jemal A. Cancer statistics, 2017. CA Cancer J Clin. 2017; 67(1):7-30.

3. Rahib L, Smith BD, Aizenberg R, Rosenzweig AB, Fleshman JM, Matrisian LM. Projecting cancer incidence and deaths to 2030: the unexpected burden of thyroid, liver, and pancreas cancers in the United States. Cancer Res. 2014; 74:2913-21.

4. Wang $S$, Huang $S$, Sun YL. Epithelial-Mesenchymal transition in pancreatic cancer: a review. Biomed Res Int. 2017;2017:2646148.

5. Liu Q, Sheng W, Dong M, Dong X, Dong Q, Li F. Gli1 promotes transforming growth factor-beta1- and epidermal growth factor-induced epithelial to mesenchymal transition in pancreatic cancer cells. Surgery. 2015;158(1):211-24.

6. Kudinov AE, Karanicolas J, Golemis EA, Boumber Y. Musashi RNA-binding proteins as Cancer drivers and novel therapeutic targets. Clin Cancer Res. 2017;23(9):2143-53.

7. Park SM, Deering RP, Lu Y, Tivnan P, Lianoglou S, Al-Shahrour F, et al. Musashi-2 controls cell fate, lineage bias, and TGF-b signaling in HSCs. J Exp Med. 2014;211(1):71-87.

8. Sakakibara S, Nakamura Y, Yoshida T, Shibata S, Koike M, Takano H, Ueda S, Uchiyama Y, Noda T, Okano H. RNA-binding protein Musashi family: roles 
for CNS stem cells and a subpopulation of ependymal cells revealed by targeted disruption and antisense ablation. Proc Natl Acad Sci U S A. 2002; 99:15194-9.

9. Wang S, Li N, Yousefi M, Nakauka-Ddamba A, Li F, Parada K, Rao S, Minuesa $G$, et al. Transformation of the intestinal epithelium by the MSI2 RNAbinding protein. Nat Commun. 2015;6:6517.

10. Byers RJ, Currie T, Tholouli E, Rodig SJ, Kutok JL. MSI2 protein expression predicts unfavorable outcome in acute myeloid leukemia. Blood. 2011;118:2857-67.

11. Ito T, Kwon HY, Zimdahl B, Congdon KL, Blum J, Lento WE, Zhao C, Lagoo $A$, et al. Regulation of myeloid leukaemia by the cell fate determinant Musashi. Nature. 2010;466:765-8.

12. Cox JL, Wilder PJ, Gilmore JM, Wuebben EL, Washburn MP, Rizzino A. The SOX2-interactome in brain cancer cells identifies the requirement of MSI2 and USP9X for the growth of brain tumor cells. PLoS One. 2013;8(5):e62857.

13. He L, Zhou X, Qu C, Hu L, Tang Y, Zhang Q, Liang M, Hong J. Musashi2 predicts poor prognosis and invasion in hepatocellular carcinoma by driving epithelial-mesenchymal transition. J Cell Mol Med. 2014;18(1):49-58.

14. Emadi-Baygi M, Nikpour P, Mohammad-Hashem F, Maracy MR, HaghjooyJavanmard S. MSI2 expression is decreased in grade II of gastric carcinoma. Pathol-Res Pract. 2013;209:689-91.

15. Yang C, Zhang W, Wang L, Kazobinka G, Han X, Li B, Hou T. Musashi-2 promotes migration and invasion in bladder cancer via activation of the JAK2/STAT3 pathway. Lab Invest. 2016;96(9):950-8.

16. Sheng W, Dong M, Chen C, Li Y, Liu Q, Dong Q. Musashi2 promotes the development and progression of pancreatic cancer by down-regulating numb protein. Oncotarget. 2017;8(9):14359-73.

17. Sheng W, Dong M, Chen C, Wang Z, Li Y, Wang K, Li Y, Zhou J. Cooperation of Musashi-2, numb, MDM2, and P53 in drug resistance and malignant biology of pancreatic cancer. FASEB J. 2017:31(6):2429-38.

18. Sheng W, Chen C, Dong M, Wang G, Zhou J, Song H, Li Y, Zhang J, Ding S. Calreticulin promotes EGF-induced EMT in pancreatic cancer cells via integrin/ EGFR-ERK/MAPK signaling pathway. Cell Death Dis. 2017;8(10):e3147.

19. Sheng W, Dong M, Zhou J, et al. Tumor size and clinical stage are independent risk predictors for the high occurrence and poor prognosis of postoperative liver metastasis in patients with radically resectable pancreatic cancer. Int J Clin Exp Pathol. 2016;9(2):854-65.

20. Caramel J, Ligier M, Puisieux A. Pleiotropic roles for ZEB1 in Cancer. Cancer Res. 2018;78(1):30-5.

21. Xie L, Law BK, Chytil AM, Brown KA, Aakre ME, Moses HL. Activation of the Erk pathway is required for TGF-beta1-induced EMT in vitro. Neoplasia. 2004; 6(5):603-10.

22. Navandar M, Garding A, Sahu SK, Pataskar A, Schick S, Tiwari VK. ERK signalling modulates epigenome to drive epithelial to mesenchymal transition. Oncotarget. 2017;8(17):29269-81.

23. Cho KB, Cho MK, Lee WY, Kang KW. Overexpression of c-myc induces epithelial mesenchymal transition in mammary epithelial cells. Cancer Lett. 2010;293(2):230-9.

24. Li NY, Weber CE, Wai PY, Cuevas BD, Zhang J, Kuo PC, Mi Z. An MAPKdependent pathway induces epithelial-mesenchymal transition via twist activation in human breast cancer cell lines. Surgery. 2013;154(2):404-10

25. Amatangelo MD, Goodyear S, Varma D, Stearns ME. C-Myc expression and MEK1-induced Erk2 nucleus localization are required for TGF-beta induced epithelial-mesenchymal transition and invasion in prostate cancer. Carcinogenesis. 2012;33(10):1965-75.

26. Chiu LY, Hsin IL, Yang TY, Sung WW, Chi JY, Chang JT, Ko JL, Sheu GT. The ERK-ZEB1 pathway mediates epithelial-mesenchymal transition in pemetrexed resistant lung cancer cells with suppression by vinca alkaloids. Oncogene. 2017;36(2):242-53.

27. Kurahara H, Takao S, Maemura K, Mataki Y, Kuwahata T, Maeda K, Ding Q Sakoda M, et al. Epithelial-mesenchymal transition and mesenchymalepithelial transition via regulation of ZEB-1 and ZEB-2 expression in pancreatic cancer. J Surg Oncol. 2012;105(7):655-61.

28. Javle MM, Gibbs JF, Iwata KK, Pak Y, Rutledge P, Yu J, Black JD, Tan D, Khoury T. Epithelial-mesenchymal transition (EMT) and activated extracellular signal-regulated kinase (p-Erk) in surgically resected pancreatic cancer. Ann Surg Oncol. 2007:14(12):3527-33.

29. Schleger C, Verbeke C, Hildenbrand R, Zentgraf H, Bleyl U. C-MYC activation in primary and metastatic ductal adenocarcinoma of the pancreas: incidence, mechanisms, and clinical significance. Mod Pathol. 2002;15(4):462-9.
30. Han Y, Ye A, Zhang Y, Cai Z, Wang W, Sun L, et al. Musashi-2 silencing exerts potent activity against acute myeloid leukemia and enhances Chemosensitivity to Daunorubicin. PLoS One. 2015;10(8):e0136484.

31. Fang T, Lv H, Wu F, Wang C, Li T, Lv G, Tang L, Guo L, Tang S, Cao D, Wu M, Yang W, Wang H. Musashi 2 contributes to the stemness and chemoresistance of liver cancer stem cells via LIN28A activation. Cancer Lett. 2017;384:50-9.

32. Kang MH, Jeong KJ, Kim WY, Lee HJ, Gong G, Suh N, Győrffy B, Kim S, et al. Musashi RNA-binding protein 2 regulates estrogen receptor 1 function in breast cancer. Oncogene. 2017;36(12):1745-52.

33. Hu F, Liu C, Xie F, Lin X, Yang J, Wang C, Huang Q. MSI2 knockdown represses extrahepatic cholangiocarcinoma growth and invasion by inhibiting EMT. Onco Targets Ther. 2018;11:4035-46.

34. Li Z, Jin H, Mao G, Wu L, Guo Q. Msi2 plays a carcinogenic role in esophageal squamous cell carcinoma via regulation of the $\mathrm{Wnt} / \mathrm{\beta}$-catenin and hedgehog signaling pathways. Exp Cell Res. 2017;361(1):170-7.

35. Kudinov AE, Deneka A, Nikonova AS, Beck TN, Ahn YH, Liu X, Martinez CF, Schultz FA, et al. Musashi-2 (MSI2) supports TGF- $\beta$ signaling and inhibits claudins to promote non-small cell lung cancer (NSCLC) metastasis. Proc Natl Acad Sci U S A. 2016;113(25):6955-60.

36. Lazarova D, Bordonaro M. ZEB1 mediates drug resistance and EMT in p300deficient CRC. J Cancer. 2017:8(8):1453-9.

37. Krebs AM, Mitschke J, Lasierra Losada M, Schmalhofer O, Boerries M, Busch $\mathrm{H}$, Boettcher M, Mougiakakos D, et al. The EMT-activator Zeb1 is a key factor for cell plasticity and promotes metastasis in pancreatic cancer. Nat Cell Biol. 2017:19(5):518-29.

38. Preca BT, Bajdak K, Mock K, Lehmann W, Sundararajan V, Bronsert P, MatzgeOgi A, et al. A novel ZEB1/HAS2 positive feedback loop promotes EMT in breast cancer. Oncotarget. 2017;8(7):11530-43.

39. Larsen JE, Nathan V, Osborne JK, Farrow RK, Deb D, Sullivan JP, Dospoy PD, Augustyn A, et al. ZEB1 drives epithelial-to-mesenchymal transition in lung cancer. J Clin Invest. 2016;126(9):3219-35.

40. Zhang $Y, X u L, L i A, \operatorname{Han} X$. The roles of $Z E B 1$ in tumorigenic progression and epigenetic modifications. Biomed Pharmacother. 2019;110:400-8.

41. Yin S, Cheryan VT, Xu L, Rishi AK, Reddy KB. Myc mediates cancer stem-like cells and EMT changes in triple negative breast cancers cells. PLoS One. 2017:12(8):e0183578

42. Li H, Jin Y, Hu Y, Jiang L, Liu F, Zhang Y, Hao Y, Chen S, Wu X, Liu Y. The PLGF/C-MYC/miR-19a axis promotes metastasis and stemness in gallbladder cancer. Cancer Sci. 2018 May; 109(5):1532-44.

43. Bae GY, Choi SJ, Lee JS, Jo J, Lee J, Kim J, Cha HJ. Loss of E-cadherin activates EGFR-MEK/ERK signaling, which promotes invasion via the ZEB1/ MMP2 axis in non-small cell lung cancer. Oncotarget. 2013;4(12):2512-22.

44. Zhang MR, Xi S, Shukla V, Hong JA, Chen H, Xiong Y, Ripley RT, Hoang CD, Schrump DS. The Pluripotency Factor Musashi-2 Is a Novel Target for Lung Cancer Therapy. Ann Am Thorac Soc. 2018;15(Supplement_2):S124.

45. MC MN, Cragle CE, FK MD, Hardy LL, Wang Y, Arumugam K, Rahmatallah Y, Glazko GV. Evasion of regulatory phosphorylation by an alternatively spliced isoform of Musashi2. Sci Rep. 2017;7(1):11503.

46. Minuesa G, Albanese SK, Xie W, Kazansky Y, Worroll D, Chow A, Schurer A, Park S-M, et al. Small-molecule targeting of MUSASHI RNA-binding activity in acute myeloid leukemia. Nat Commun. 2019;10:2691.

\section{Publisher's Note}

Springer Nature remains neutral with regard to jurisdictional claims in published maps and institutional affiliations.

\section{Ready to submit your research? Choose BMC and benefit from:}

- fast, convenient online submission

- thorough peer review by experienced researchers in your field

- rapid publication on acceptance

- support for research data, including large and complex data types

- gold Open Access which fosters wider collaboration and increased citations

- maximum visibility for your research: over $100 \mathrm{M}$ website views per year

At $\mathrm{BMC}$, research is always in progress.

Learn more biomedcentral.com/submissions 